\title{
Performance Evaluation of the PRMA Protocol for Voice and Data Transmissions in Low Earth Orbit Mobile Satellite Systems
}

\author{
Enrico Del Re*, Romano Fantacci*, Giovanni Giambene** \\ * Dipartimento di Ingegneria Elettronica - Università degli Studi di Firenze \\ Via S. Marta, 3 - 50139 Firenze, ITALY
}

** OTE S.p.A., Via E. Barsanti, 8, 50127 Firenze, ITALY

\begin{abstract}
The Universal Mobile Telecommunications System (UMTS) will provide multimedia services to users in whatever environment via uniform service access procedures. Efficient Medium Access Control (MAC) protocols are needed to guarantee a high capacity. This paper deals with Packet Reservation Multiple Access (PRMA), widely considered as an efficient MAC protocol in terrestrial microcellular systems, for voice and data transmissions in a UMTS based on Low Earth Orbit - Mobile Satellite Systems (LEO-MSSs). Through a suitable choice of the system parameter values, the PRMA protocol may achieve a good quality of service also in LEO-MSSs.
\end{abstract}

\section{Introduction}

The Universal Mobile Telecommunications System (UMTS) will provide a wide range of services to subscribers by integrating a terrestrial component and a satellite component [1]. Presently implemented Mobile Satellite Systems (MSSs) provide only low bitrate data applications or higher bit-rates, but for fixed terminals [2]. Therefore, none of the these systems meets the requirements of UMTS which is expected to provide mobile users with the same services and, possibly, with the same Quality of Service (QoS) as that of the terrestrial B-ISDN network based on Asynchronous Transfer Mode (ATM). ATM encompasses five traffic classes [3]:

- Constant Bit-Rate (CBR);

- Variable Bit-Rate, Real Time (VBR-RT);

- Variable Bit-Rate, Non-Real Time (VBR-NRT);

- Available Bit-Rate (ABR);

- Unspecified Bit-Rate (UBR).

Within UMTS, broadband-MSSs will aim at supporting mainly VBR-RT, VBR-NRT and ABR traffics [3]. Moreover, Low Earth Orbit - Mobile Satellite Systems (LEO-MSSs) [2] will play a fundamental role in the satellite segment of UMTS.

This paper proposes the Packet Reservation Multiple Access protocol (PRMA) as an efficient Medium
Access Control (MAC) protocol in LEO-MSSs. The PRMA scheme is an improvement of Time Division Multiple Access (TDMA) that combines TDMA with Slotted-ALOHA [4]. PRMA was initially proposed and investigated in terrestrial microcellular systems characterized by a negligible Round Trip Delay (RTD) as regards the slot duration [4]. RTD represents the time a UT must wait before knowing the outcome of its transmission attempt. It has been found that PRMA can efficiently manage both voice and data traffics [5]. The main obstacle for applying the PRMA protocol to MSSs is the high RTD value. Some initial studies have highlighted that the RTD value of geostationary satellite systems (i.e., minimum $250 \mathrm{~ms}$ ) prevents any application of PRMA [6]. Conversely, this paper shows that PRMA may efficiently support both voice VBR-RT traffic and data (ABR-like) traffic in LEOMSSs, where RTD ranges from $10 \mathrm{~ms}$ to $30 \mathrm{~ms}$.

2. The PRMA protocol in LEO-MSSs: voice and data applications

This paper assumes a LEO-MSS where cells are fixed on the earth: satellite antenna spot-beams are steered to point to the same area on the earth as long as possible [7]. A given cell of the MSS is considered, where the UTs share a carrier to transmit their packets to the related satellite. We consider UTs requesting voice calls (i.e., voice UTs) and UTs requesting data transmissions (i.e., data UTs): they belong to two sub-systems with different characteristics for both the packet generation process and the required QoS.

When a voice or data UT needs to transmit packets, it enters the contending state (CON) [4] to obtain the reservation of a slot per frame: as soon as there is an available slot and the UT has the permission, it transmits its first packet. Permission is generated at each contending UT with a specified probability and permissions at different UTs are independent. In order to fulfill the different QoS requirements for voice and data traffics, different permission probabilities $p_{v}, p_{d}$ have been assigned to voice and data, respectively.

If the transmission attempt of a UT is successful (i.e., the header of the packet sent on a given slot has been 
correctly decoded at the satellite), the slot is devoted in each subsequent frame for the exclusive use of that UT. The satellite broadcasts feedback packets to inform all the UTs of a cell about the state of each slot of the PRMA carrier.

If two or more UTs have attempted to send their packets on the same slot, there is a collision. If we neglect the capture effect [4], the satellite can not recognize any UT. Therefore, the involved UTs reschedule randomly their transmission attempts on free slots, according to the contending procedure.

In LEO-MSSs, RTD depends on the satellite constellation altitude, the minimum elevation angle envisaged for UTs and the relative UT-satellite disposition. Moreover, RTD usually varies during call lifetime mainly due to the satellite constellation dynamics. Hence, we have assumed RTD always equal to its maximum value for a given constellation, $R T D_{\max }$ (conservative assumption). Let $T_{f}$ denote the frame duration and $T_{s}$ the slot duration. We have selected $T_{f}=$ $R T D_{\max }+\varepsilon^{1}$. This means that a UT is informed about the outcome of a transmission attempt at the beginning of the homologous slot in the subsequent frame. We have assumed that a UT can perform only one access attempt per frame.

\section{Voice sub-system}

Each voice packet to be transmitted is formed by the bits produced by a voice source in $T_{f}$ plus a header with $H_{v}$ bits. Hence, we have that the number of slots per frame, $N_{s}$, and the slot duration, $T_{s}$, are:

$$
N_{s}=\left\lfloor\frac{R_{c} T_{f}}{R_{s} T_{f}+H_{v}}\right\rfloor \quad\left[\frac{\text { slots }}{\text { frame }}\right], T_{s}=\frac{T_{f}}{N_{s}}[\mathrm{~ms}]
$$

where $\lfloor x\rfloor$ denotes the biggest integer number $\leq x$.

We have assumed a slow Voice Activity Detector (VAD) [4] which reveals only principal gaps within a conversation. This model has been investigated by OTE S.p.A. for the VAD to be used for effective handling of PABX ISDN calls in the development of the TErrestrial Truncked RAdio (TETRA) system [8].

Note that a fast VAD [4] can not attain a good performance in an MSS, since it entails a greater contention rate, which has to be avoided in the

\footnotetext{
${ }^{1}$ The satellite recognizes the UT requesting a reservation on a given slot on the basis of the header of the packet received; $\varepsilon$ is the time to transmit the packet header. When the header is decoded, the satellite broadcasts a suitable acknowledgement on the feedback channel. If $T_{f}=R T D_{m a x}+\varepsilon$, the UT requesting a reservation on a given slot will know the outcome of its attempt before the homologous slot in the subsequent frame. Practically, $\varepsilon$ can be neglected as regards $R T D_{\max }$; hence $T_{f} \approx R T D_{\max }$.
}

presence of high RTD values. On the basis of the slow VAD, a conversation is divided in talking phases and silent phases; the times spent in these states have been modeled by exponential distributions with expected values $1 \mathrm{~s}$ and $1.35 \mathrm{~s}$, respectively, for the talking phase and the silent phase.

A voice $U T$ in the CON state discards the first packet from the buffer when the time to obtain a reservation exceeds a maximum value, $D_{\max }$. We have assumed $D_{\max }$ equal to $32 \mathrm{~ms}$ and, hence, we have considered that acceptable values of the voice packet dropping probability $\left(P_{\text {drop }}\right)$ must be less than or equal to $1 \%$ [4]. Let $N_{v \max }$ denote the maximum number of voice UTs with a call in progress that may share a PRMA carrier with $P_{\text {drop }} \leq 1 \% ; N_{v \max }$ depends on the parameters of both the voice sub-system and the data sub-system.

\section{Data sub-system}

We focus here on an ABR-like data traffic which is suitable for modeling e-mail messages and data file transfer. We consider $N_{d}$ data UTs which produce messages according to independent Poisson processes with mean rates $\lambda$. Every message has a random length and is segmented in packets, each with a header with $H_{d}$ bits. Let $L_{b}$ denote the average length in bits of a message (only information part); the corresponding average number of packets $L_{s}$, is given by:

$$
L_{s}=\frac{L_{b}}{R_{s} T_{f}} \quad\left[\frac{p k t s}{m s g}\right]
$$

The number of packets of a message has been assumed geometrically distributed with expected value $L_{s}$. The average input data traffic on the PRMA carrier due to all data UTs is given by:

$$
r_{d}=\lambda T_{s} L_{s} N_{d} \quad\left[\frac{p k t s}{s l o t}\right]
$$

When a data UT needs to transmit a message it must obtain the reservation of a slot per frame, as for voice packet transmissions by active voice UTs. The reservation is maintained by the data UT until it transmits all messages in the buffer (exhaustive policy). The parameter that measures the performance of the data sub-system is the average message transmission time, $T_{m s g}$, i.e., the mean time between the message arrival to the buffer of a UT and the instant when all packets of the message have been transmitted.

\section{Simulation results}

The system parameter values are given in Table 1 [5]. 


\begin{tabular}{||c|c|c|}
\hline Parameter & Definition & Value \\
\hline$R_{c}$ & channel bit rate & $720 \mathrm{kbit} / \mathrm{s}$ \\
\hline$R_{s}$ & speech source bit rate & $32 \mathrm{kbit} / \mathrm{s}$ \\
\hline$H_{v}=H_{d}$ & header size of a packet & $64 \mathrm{bit}$ \\
\hline
\end{tabular}

Table 1: System parameter values.

Fig. 1 shows numerical results concerning $P_{\text {drop }}$ and $T_{m s g}$ as a function of $r_{d}$ for $T_{f}=R T D_{\max }=16 \mathrm{~ms}, N_{v}=$ $N_{d}=15 \mathrm{UTs} /$ carrier, $p_{\nu}=p_{d}=0.5, L_{b}=25 \mathrm{kbit} / \mathrm{msg}$. It is evident from Fig. 1 that the maximum $r_{d}$ value that can be supported by fulfilling $P_{\text {drop }} \leq 1 \%$ is 0.47 pkts/slot; correspondingly, the voice sub-system throughput is $0.31 \mathrm{pkts} / \mathrm{slot}$ and $T_{\mathrm{msg}}$ is about 2700 slots. This performance can be improved by a suitable selection of system parameter values (i.e., $p_{v}, p_{d}$ and $T_{f}$ ), as shown in Section 3.
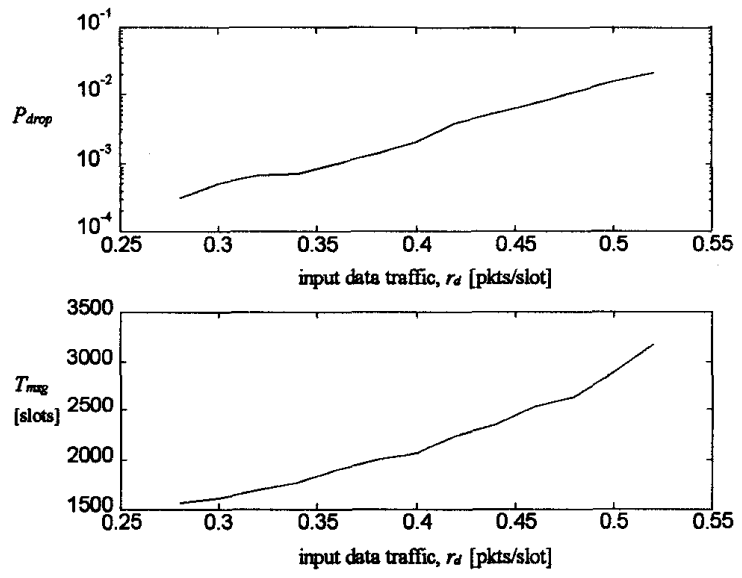

Fig. 1: Behavior for both $P_{\text {drop }}$ and $T_{m s g}$ as a function of $r_{d}$ $\left(T_{f}=R T D_{\max }=16 \mathrm{~ms}, N_{v}=N_{d}=15 \mathrm{UTs} /\right.$ carrier, $p_{v}=p_{d}=$ $0.5, L_{b}=25 \mathrm{kbit} / \mathrm{msg}$ ).

\section{Selection of system parameters}

The criterion adopted to select the most suitable values for both $p_{\nu}, p_{d}$ and $T_{f}$ is that of minimizing $P_{\text {drop }}$ without significantly increasing $T_{m s g}$. Fig. 2 shows $P_{\text {drop }}$ as a function of both $p_{v}$ and $p_{d}$. The numerical values have been obtained through computer simulations with $T_{f}=R T D_{\max }=16 \mathrm{~ms}, N_{v}=N_{d}=15 \mathrm{UTs} /$ carrier, $r_{d}=$ $0.4 \mathrm{pkts} / \mathrm{slot}, L_{b}=25 \mathrm{kbit} / \mathrm{msg}$. In Fig. 2, we note that:

- $P_{\text {drop }}$ mainly depends on $p_{v}$. When $p_{v}$ is too low, $P_{\text {drop }}$ increases due to the growth of the access delay to obtain a reservation. Moreover, if $p_{v}$ is close to 1 , $P_{\text {drop }}$ increases, because collisions are more frequent among the attempts of UTs which need to transmit their talkspurts.
- $P_{\text {drop }}$ increases with $p_{d}$; if $p_{d}$ increases more data UTs attempt their transmissions on available slots, thus hindering the reservation of voice UTs. An excessive reduction of $p_{d}$ must be avoided to prevent high values of $T_{m s g}$.

From Fig. 2 it appears that $p_{v}=0.6$ is the best choice. Moreover, we have verified that $T_{m s g}$ is practically independent of $p_{v}$ and $p_{d}$, if $p_{d} \geq 0.2$. Whereas, if $p_{d}<$ $0.2, T_{\text {msg }}$ suddenly increases when $p_{d}$ decreases. Hence, $p_{d}=0.2$ is a good choice that guarantees low $P_{\text {drop }}$ values without unacceptably increasing $T_{\text {msg. }}$. We have obtained the same optimized values for both $p_{v}$ and $p_{d}$ with different values of $T_{f}=R T D_{\max }, r_{d}$ and $L_{b}$.

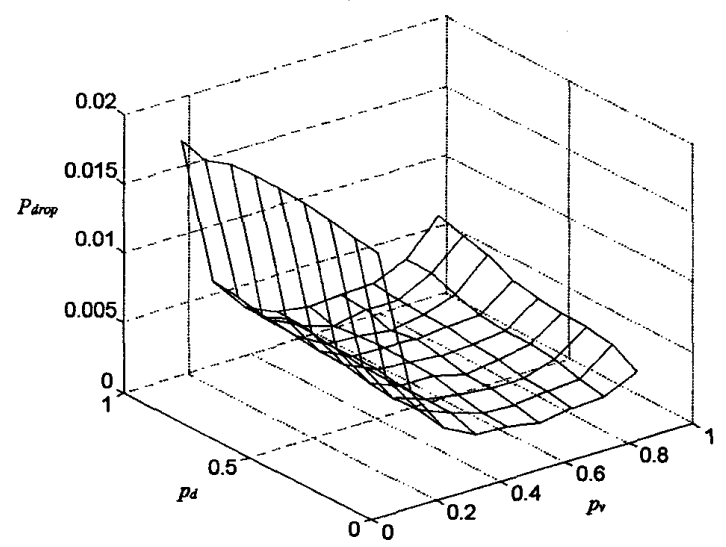

Fig. 2: $P_{d r o p}$ performance as a function of both $p_{v}$ and $p_{d}\left(T_{f}\right.$ $=R T D_{\max }=16 \mathrm{~ms}, N_{v}=N_{d}=15 \mathrm{UTs} /$ carrier, $r_{d}=0.4$ $\mathrm{pkt} / \mathrm{slot}, L_{b}=25 \mathrm{kbit} / \mathrm{msg}$ ).

Let us focus on the selection of the optimum value for $T_{f}$. By assuming fixed values for $R_{c}, R_{s}, H_{v}=H_{d}, L_{b}$, if $T_{f}$ increases, the average number of packets per talkspurt as well as the average number of packets per message decreases; whereas, according to (1), $N_{s}$ increases up to the maximum value $\left\lfloor R_{c} / R_{s}\right\rfloor$, which is equal to 22 slots/frame for the values in Table 1 . Parameter $T_{m s g}$ slightly depends on $T_{f}=R T D_{\max }$ in LEO-MSSs: a high $T_{f}$ value allows a slight increase of $N_{s}$ that, in turn, increases the transmission attempt rate. While, a variation of $T_{f}$ has a greater impact on $P_{\text {drop }}$ :

- an excessive $T_{f}$ value causes a high $P_{\text {drop }}$ value due to the maximum acceptable delay $D_{\max }$ : a reduced number of attempts is available for the contending voice UTs to obtain a reservation; many packets experience an access delay greater than $D_{\text {max }}$.

- With a low value of $T_{f}$, few slots are available per frame for the contention of the active UTs. This leads to an increase in the mean number of UTs in the CON state and, hence, to high $P_{\text {drop }}$ values. 
The optimum $T_{f}$ value is that which allows the maximum capacity of voice UTs per PRMA carrier (i.e., the maximum value of $N_{v \max }$ ). We use the optimum permission probabilities obtained at the previous step. Fig. 3 presents the behavior of $N_{v \max }$ as a function of $T_{f}$ with $p_{v}=0.6, p_{d}=0.2, N_{d}=15$ data UTs $/$ carrier, $r_{d}=0.4 \mathrm{pkts} / \mathrm{slot}, L_{b}=25 \mathrm{kbit} / \mathrm{msg}$. $N_{v \max }$ has a maximum equal to 18 voice UTs/carrier for $T_{f}$ ranging from 16 to $19 \mathrm{~ms}$; we have selected $T_{f}=16 \mathrm{~ms}$ to be consistent with [4], where terrestrial microcellular systems are considered. Therefore, the optimization of $T_{f}$ is quite insensitive to the RTD value, provided that RTD belongs to the LEO range.

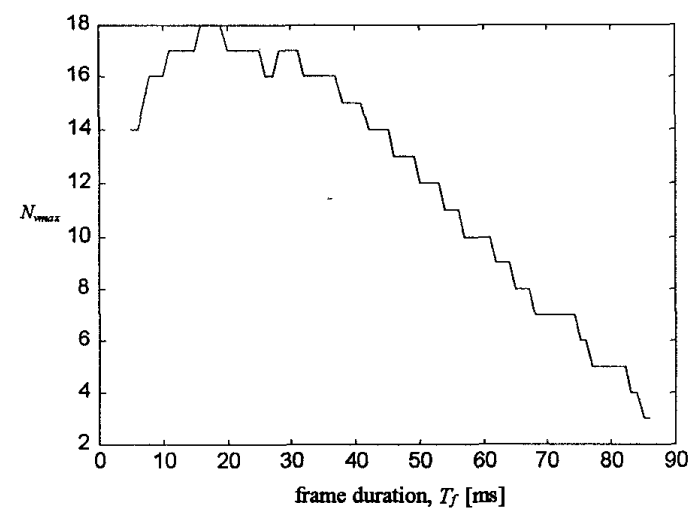

Fig. 3: Behavior of $N_{v \max }$ as a function of $T_{f}\left(p_{f}=0.6, p_{d}=\right.$ $0.2, N_{d}=15$ data UTs/carrier, $r_{d}=0.4 \mathrm{pkt} / \mathrm{slot}, L_{b}=25$ kbit/msg).

Let us focus on the multiplexing gain achievable for the voice sub-system by PRMA, $\mu_{v}$; this parameter is defined as the ratio between the maximum number of voice UTs supported per PRMA carrier, $N_{\text {vmax }}$, and the equivalent number of TDMA channels devoted to voice UTs (an ideal TDMA without packet overhead is considered). The number of TDMA slots is given by $\left\lfloor R_{c} / R_{s}\right\rfloor$, but only a part of these slots will be used by voice UTs in the equivalent ideal TDMA for the voice sub-system: $\lambda L_{b} N_{d}$ bit/s of the total channel capacity $R_{c}$ are on average used for data transmissions. Therefore, the number of equivalent TDMA channels is $\left\lfloor\left(R_{c}-\lambda L_{b} N_{d}\right) / R_{s}\right\rfloor$. In conclusion, $\mu_{v}$ is given by:

$$
\mu_{v}=\frac{N_{v \max }}{\left[\frac{R_{c}-\lambda L_{b} N_{d}}{R_{s}}\right]} \quad\left[\frac{\text { voice conversations }}{\text { voice channel }}\right]
$$

Fig. 4 shows the behavior of $\mu_{v}$ as a function of $r_{d}$ for $L_{b}=25 \mathrm{kbit} / \mathrm{msg}, N_{d}=20$ data UTs $/$ carrier and the selected optimum parameters (i.e., $p_{v}=0.6, p_{d}=0.2$, $\left.T_{f}=16 \mathrm{~ms}\right)$. We can note that $\mu_{v}$ decreases as $r_{d}$ increases. This behavior can be justified as follows: $\mu_{v}$ depends on the number of slots per frame to support voice transmissions; when $r_{d}$ increases, we have a reduced number of slots to be devoted to voice transmissions and, therefore, a low $\mu_{\nu}$ value. We have $\mu_{v} \geq 1$ for $r_{d} \leq 0.6$ data pkts/slot. In these conditions, PRMA allows a more efficient management of voice sources than TDMA. Moreover, when $r_{d}=0$ (i.e., no data traffic) we obtain $\mu_{v} \approx 1.6$ voice conversations/voice channel, i.e., the same $\mu_{v}$ value of terrestrial microcellular systems with only voice sources and optimized parameter values [4].

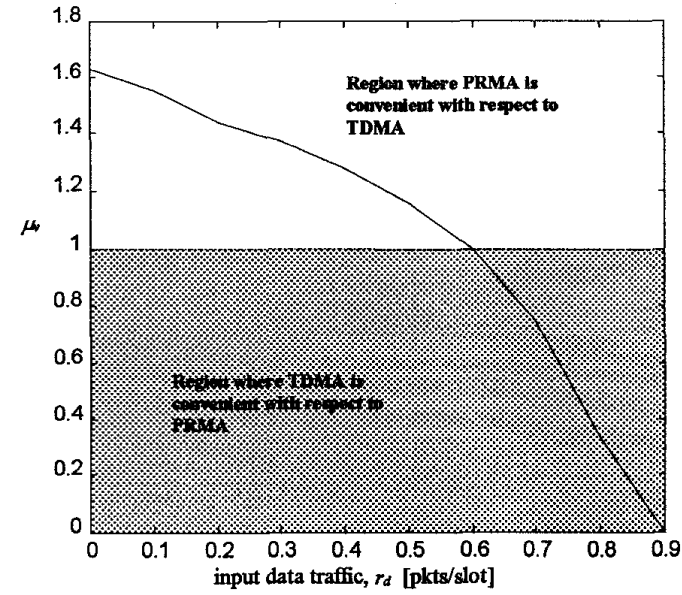

Fig. 4: Behavior of $\mu_{v}$ as a function of $r_{d}\left(L_{b}=25 \mathrm{kbit} / \mathrm{msg}\right.$, $N_{d}=20$ data UTs/carrier, $p_{v}=0.6, p_{d}=0.2, T_{f}=16 \mathrm{~ms}$ ).

Since a given $r_{d}$ value can be obtained either with short messages and a high arrival rate or with long messages and a low arrival rate, we evaluate in Fig. 5 the impact of different message lengths $L_{b}$ for $r_{d}=0.4$ data pkts/slot, $N_{v}=N_{d}=15 \mathrm{UTs} /$ carrier and optimized system parameter values (i.e., $p_{v}=0.6, p_{d}=0.2, T_{f}=$ $16 \mathrm{~ms})$. Two opposite trends are found in Fig. 5 :

- when $L_{b}$ is low, $P_{\text {drop }}$ increases, because we have a high arrival rate of messages. It is likely that the service of a message ends before a new message needs to be sent by the same data UT. Hence, the contention rate is high and causes a worse performance of the voice sub-system.

- If $L_{b}$ is high, $P_{\text {drop }}$ increases, because the transmission of the message of a UT requires a long time; during this interval, new messages may arrive at the buffer of the UT and the slot reservation at the end of the present message is not released (exhaustive policy). Therefore, $P_{\text {drop }}$ increases because the number of slots available for UTs with new talkspurts is practically reduced. 

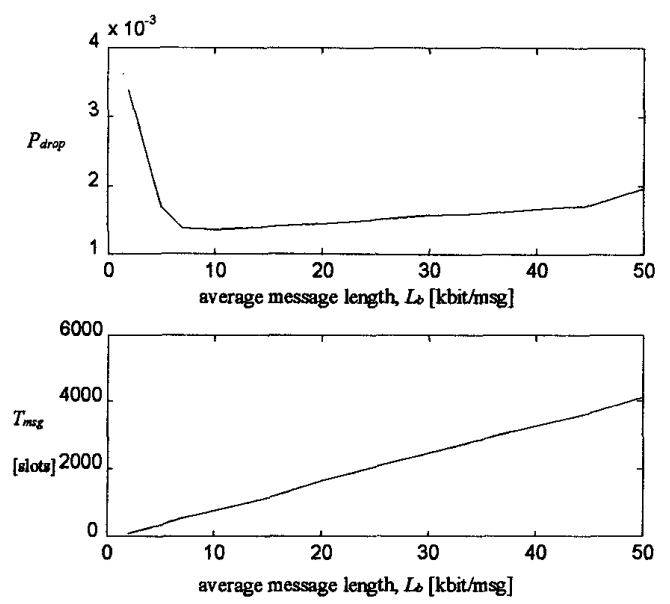

Fig. 5: Impact of different message lengths $L_{b}\left(r_{d}=0.4\right.$ data pkts/slot, $N_{v}=N_{d}=15$ UTs/carrier, $p_{v}=0.6, p_{d}=0.2$, $\left.T_{f}=16 \mathrm{~ms}\right)$.

\section{Comparison between terrestrial microcellular systems and LEO-MSSs}

We compare the PRMA protocol performance in a terrestrial microcellular system and in a LEO-MSS to evaluate the impact of RTD on both $P_{\text {drop }}$ and $T_{m s g}$. In a terrestrial microcellular system, the RTD can be neglected with respect to $T_{s}$ : a UT attempting a transmission on a free slot immediately receives the outcome of its transmission from the base station of its cell. If the attempt has been unsuccessful the UT can reschedule the transmission even from the next slot, provided that both this slot is available and the UT attains the permission to transmit. Fig. 6 shows the behavior of $P_{d r o p}$ and $T_{m s g}$ as a function of $r_{d}$ for both a terrestrial system and a LEO-MSS.
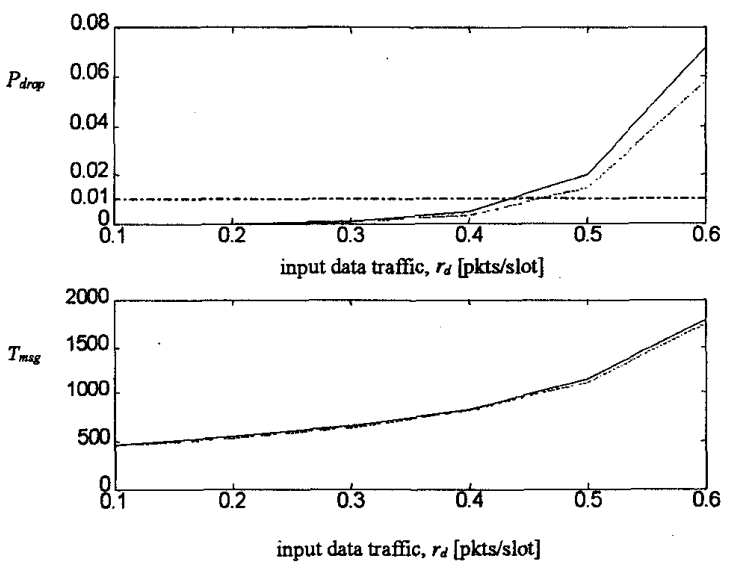

Fig. 6: Behavior of $P_{d r o p}$ and $T_{m s g}$ as a function of $r_{d}$, in a terrestrial microcellular system, dashed line, and in a LEO-MSS, continuous line $\left(T_{f}=16 \mathrm{~ms}, N_{v}=N_{d}=16\right.$ $\mathrm{UTs} /$ carrier, $p_{v}=0.35, p_{d}=0.15, L_{s}=20 \mathrm{pkts} / \mathrm{msg}$ ).
In Fig. 6, the differences between the two situations are small, if $P_{\text {drop }} \leq 1 \%$ : the maximum $r_{d}$ value is 0.45 $\mathrm{pkts} / \mathrm{slot}$ in the terrestrial case, and $0.43 \mathrm{pkts} / \mathrm{slot}$ in the LEO-MSS case. Correspondingly, parameter $T_{m s g}$ is on the order of hundreds of slots with a difference of few tens of slots (practically, RTD) between the satellite case and the terrestrial one. Hence, the PRMA protocol manages voice and data traffics in LEO-MSSs with a QoS very close to that obtained in terrestrial systems.

\section{Conclusions}

This paper has studied the extension of the PRMA protocol to LEO-MSSs. In particular, we have focused on the VBR-RT voice service and an ABR-like data service. The PRMA performance has been evaluated and optimized by simulations. The obtained results clearly point out that PRMA is advantageous with respect to TDMA in managing voice and data traffics. Therefore, PRMA is a good candidate as a unified MAC protocol for both the terrestrial component and the LEO-MSS component of the future UMTS.

Acknowledgements. The authors wish to thank Ing. Carlo Cerboni for useful comments and discussions.

\section{References}

1. I. Berberana, T. Megedanz, S. Covaci, "Evolution from Existing Telecommunications Systems towards UMTS", GLOBECOM'96, London, Nov. 18-22, 1996.

2. Web sites with addresses: http://www.iridium.com, http://globalstar.com, http://www.teledesic.com.

3. V. Leung, H. Alnuweiri, and P. Nasiopoulos, "Interworking Broadband Satellite Networks with terrestrial Networks Subsystems", University of British Columbia - Internal Report.

4. S. Nanda, D. J. Goodman, U. Timor, "Performance of PRMA: a Packet Voice Protocol for Cellular Systems", IEEE Trans. Veh. Tech., Vol. 40, No. 3, pp. 584-598, Aug. 1991.

5. W. C. Wong, D. J. Goodman, "Integrated Data and Speech Transmission using Packet Reservation Multiple Access", ICC'94, pp. 172-176, 1994.

6. F. Ananasso, F. Delli Priscoli, "The Role of Satellites in Personal Communication Services", IEEE J. Sel. Areas Comm., Vol. 13, No. 2, pp. 180-196, Feb. 1995.

7. J. Restrepo, G. Maral, "Coverage Concepts for Satellite Constellations Providing Communications Services to Fixed and Mobile Users", Space Comm., Vol. 13, No 2, pp. 145-157, 1995.

8. "Radio Equipment and Systems (RES); TransEuropean Trunked Radio (TETRA); Voice plus data (V+D); part 1: General Network Design II", ETSI ETS 300392 - 1, Edition 1, 1996. 